https://doi.org/10.48009/1_iis_2005_231-237

\title{
RESPONDING TO THE CHALLENGE OF ACADEMIC INTEGRITY IN DISTANCE LEARNING: USING EXCEL TO GUARANTEE INDIVIDUAL EFFORT
}

\author{
Paul M. Goldwater, University of Central Florida, paul.goldwater@bus.ucf.edu \\ Timothy J. Fogarty, Case Western Reserve University, tjf@ case.edu
}

\begin{abstract}
The distance learning environment is highly dependent upon assignment-based assessment. Whereas the business academy may seem well prepared for this transition with the emergence of the case method, a primary objection has been unanswered. Cases generate "canned" solutions that, when passed between students, jeopardize the accountability of individual efforts and the educational value of the exercise. Although students have leveraged computer technology to exacerbate this problem, the professoriate generally has not "fought fire with fire." Through the use of artificial intelligence can restore the confidence that each student will work his/her own case solution and will therefore extract the intended educational purpose from the effort. With computer technology made to act intelligently, assessments given to students should become more robust as a primary pedagogical device of distance learning.
\end{abstract}

Keywords: Excel, artificial intelligence, randomization, automated grading

One of the least recognized challenges of the distance learning environment pertains to the need to reconfigure student evaluation. This paper describes the problem and proposes a solution based on artificial intelligence concepts that is deliverable within conventional software.

The ability to rapidly transfer data and to instantaneously communicate simultaneously makes distance learning possible and threatens its integrity. Much more than with the "face-to-face" learning environment, instructors are heavily dependent upon assignments completed under uncontrolled circumstances. While this packaging of educational material can come closer to addressing the factual richness of the setting, they may be ineffective as measures of student ability. If instructors ignore the prospect that students exchange solutions, individual evaluation is considerably compromised. In this paper, we suggest that computer technology and artificial intelligence can be better brought to bear on the production of case materials so that academic integrity can be maintained.

\section{THE NATURE OF THE PROBLEM}

Concern over the integrity of the solution remains a legitimate concern in the traditional educational environment. Unlike tests, instructors have little control over the actual inputs of individual students when cases are a major part of the course. When cases include expectations that students produce written solutions that are to be graded, individual accountability is at issue. Notwithstanding ethical codes to the contrary, students, in the preparation of their written responses, may "borrow" solutions prepared by other students. This may occur between students in the current year's class, or through more systematic means, between students taking the class in different terms. Fraternities and sororities are notorious in the maintenance of files containing solutions to cases, as well as other materials, in order to reduce the necessary effort of each 
subsequent cohort of students. Such means of cheating may make more sensible the otherwise inexplicable finding that students spend much less time on their studies than faculty suspect [3].

The mechanisms of sharing work in the distance learning environment may vary even if the outcome does not. Students may be more widely distributed prior to and during the course they take. This lack of a relationship may reduce the psychological connection that underlies the consent of a person to share a solution with another. However, such a belief underestimates the instant intimacy of people connected through mediums such as instant messenger. Students that grow up with the normalcy of friends that they have never seen in the flesh do not believe such relationships to be of an inferior quality. In fact, lacking a physical presence may reduce the psychological risk and therefore make the relationship more intimate. To expect that less cheating will occur seems to be naïve.

A surprising few business academics have written on the regrettable fact that students will engage in cheating behaviors. Perhaps, most academics prefer not to think that this is occurring, despite the mounting evidence to the contrary [5]. Perhaps, others have little to say beyond the normative condemnation of these acts. With a few notable exceptions, academics have shunned the systematic consideration of student cheating. Anecdotally, we know that the tendency to cheat is a function of past cheating and deterrents in place, but this merely illustrates how little progress the literature has made. Meanwhile, the frequency of dishonesty in the classroom is increasing [9]. In accounting, one of the main reasons that necessitated the movement to a computerized CPA examination in 2004 was the inability to protect the paper-based examination from those that sought to cheat. Cheating may not be an ethical statement [7] but may merely reflect a reaction to the situation or opportunity. Furthermore, students seem to be quite tolerant of these behaviors among their peers [4].

While the problem of insufficient individual autonomy or accountability is present in all out-ofclass assignments, it presents special problems for cases. The essential argument for cases (the development of analytic and judgmental abilities) is undermined substantially unless the student is actively involved. Given a certain loss of efficiency inherent in the case method (i.e., less material is covered), the student who borrows case solutions may receive less of a quality business education than those exposed to a more objective question test pedagogy. Furthermore, if a non-trivial grade weight is attached to case write-ups, the integrity of evaluation also is at risk. To the extent that case solutions require spreadsheets, copying can be accomplished with negligible effort and is actually furthered by the keyboard interface of distance learning.

Instructors using digitally delivered case assignments retain some traditional methods of control. For example, visual detection can spot systematic, non-random similarities that exist between answers. This control is limited in its effectiveness for many problems whose solution depends heavily upon a small number of mathematical computations. Unlike the plethora of ways that qualitative ideas can be expressed in words, correct mathematical logic cannot differentiate, with any regularity, an independently derived path to a correct answer from the rote copying of one. In other words, only identical wrong answers look suspicious. The effectiveness of this control is also diminished when instructors delegate the grading of case materials to graduate or workstudy students who may not be as motivated to detect integrity problems. Whereas face-to-face instructors may also "cold call" students in an effort to reveal the substantive awareness beyond 
the written documentation, those running computer-mediated courses can inspect less, mostly due to the asynchronous nature of the interaction. At its best, in either medium, post hoc inspection is time-intensive, unreliable and limited in its actual effectiveness. In most cases, it does not provide substantial evidence that the academic work was not done by the student to whom it was assigned.

\section{A SOLUTION}

For as long as there have been problems, there have been incentives to cheat by finding ways, other than understanding, to produce solutions. There does not seem to be any realistic hope to "talk people out of it." Lacking this, most educators fall back on a mixture of hope and prayer and turning a blind eye to the problem. Those that proactively pursue detection have to realize that their methods are invariably a step or two behind the technology of cheating.

This paper asserts that the cheating problem lies with the nature of the problem given to students. Specifically, as long as multiple students share a problem, there will be strong incentives to share a solution. Therefore, educators who are serious about academic integrity must work to alter the problem. This paper suggests that moving toward the "cheat proof" distance learning assignment can be done using no more than the tools that we all have on our desktops.

Artificial intelligence (AI) is the field of computer science that studies how machines can be made to act intelligently. Although much has been written both for and against the ultimate achievability of true intelligence by computers, the question remains open. Articles by McDermott [6] and Woods [8] contend that understanding can be expressed in terms of symbol manipulation. They maintain that what some believe is spiritual or esoteric actually deals symbolically with "meaning rules" which represent the processing of other symbols. Suffice it to conclude that computers can emulate understanding, or at least process data as though they understand concepts within a limited scope and complexity.

Artificial intelligence systems have gained attention in many domains (e.g., health care, space exploration, computer games, etc). Business practice continues to quietly benefit from many specific expert system applications [1]. More recently, AI has been found to be effective in the business school classroom. For example, Goldwater and Fogarty [2] developed an AI system capable of generating an inexhaustible supply of multiple choice questions for student study and testing. Although not with identical technology, the computerized Certified Public Accounting examination also customizes evaluation by providing candidates with different objective question sets, equated for difficulty. A similar approach can be used to address the problem of distance learning answers that may not be independently derived.

Although artificial intelligence sounds esoteric and remote, it is readily useable so that business educators can restore the need for each student to work his/her own case solution.

Microcomputers have evolved spectacularly, so that complex systems involving AI can be processed efficiently and effectively. The basic technique used in this AI is the extraction of the symbolic representation of the case. The computer will simulate through symbols provided by the professor and duplicate the mathematical logic when formulating the case. The duplication does not have to be exact nor does it have to be at the same rate as that of the professor. In fact, a major advantage of the computer will be the speed with which it can generate the case and the solution. In essence, the computer will simulate the intelligence that was used to create the case 
and the solution. Thus, the single problem becomes a nearly infinite set of clones that are capable of illustrating the essential portions of business knowledge.

AI systems are predicated on the ability to transfer the knowledge of humans to a computer system. The computer is capable of applying that knowledge over a broader terrain than that reachable by individual human experts. In this instance, the "facts" of the case study can be operated upon via the production rules and the AI constructs that govern the development of case assignment and solution. Since many of the solutions to business problems pertain to procedures that use simple algebraic manipulation, the computations needed to produce solutions tend to be relatively straightforward.

In essence, the AI systems approach allows each student to receive a case study that has a unique set of numerical values, phrases and sentences. The alteration of certain words within the case, such as the insertion of the student's name into the cast of characters, can also be done. Each version retains the basic educational lessons of the standard case. Unique sets of values and phrases require each student to do autonomous work. If someone else's solution is submitted by a student, the answers taken will be wrong since they are in response to a question with a different set of values and phrases. Although this tailor-making could be accomplished manually by the instructor, the computational burden would be overwhelming. In any reasonably complex problem, it would be arduous to devise a unique set of numbers and sentences that obeyed the constructs necessary to allow all students to extract the same principles of decision- making with business information. However, computer processing makes this task nearly as transparently elegant as laser printing.

A large number of separate sets of hypothetical information would appear to present a grading nightmare since it would yield many equally correct answers. However, a corresponding set of solutions can be generated and matched to each individualized problem set via a common link, such as the students' university identification number. The grader uses a computer to re-perform the solution associated with that link and matches the student-provided answer to the computergenerated solution. This number also allows the instructor to provide needed assistance for a student since its input generates a case identical to the one seen by the student.

If the matching is programmed and the grading is automated via student electronic media submissions, only a reasonable amount of human work has to be involved. In a less automated environment, one could argue that the efficiency of a single "key" is thereby lost. However, such seems to be an acceptable cost for greater assurance of individual effort and accomplishment. A clear incentive exists for instructors to utilize the computer to do the grading and therefore reap the annualized return on the initial setup investment for a case that will never "go stale."

\section{DESIGN PROCESS}

Assignments are built within an Excel file comprised of four worksheets. Each worksheet represents a level in a hierarchy that builds toward the assignment seen by students. These worksheets can be called Data Verification, Assignment Validation, Iteration and Pivot Tables. Data Verification contains the actual data, the assignment wording and the algebraic functions believed necessary to the problem. Assignment Validation is the result of the data passed through from the first worksheet. At this stage, additional AI constraints are imposed upon the data 
processing. The completion of the work of the second worksheet allows the Iteration process to begin. Random number generation tagging association (with student numbers) creates 10,000 sets of numbers written to this worksheet. This process will take at least a few hours in processing work that will vary according to the complexity demanded by the algebra and the constraints. The final worksheet allows quality control to be performed. Pivot tables provide an opportunity to allow invalid and unrealistic solutions to be detected. Problems detected at this stage will necessitate programming modifications and the repeat of the four worksheet process. This section provides more detail on the elements that have been overviewed in this paragraph.

\section{Data Verification Worksheet}

Without question, accuracy at this level is the foundation of the entire design process. This worksheet depends critically upon the functional knowledge of the subject area of the designer. Most importantly, a distinction must be made between those numbers that can be changed without impact on others and those that are necessary to the structural integrity of the assignment. Forethought also has to be given to the extent to which some values are algebraically dependent upon others, either as direct mathematical results or as strong correlates. The data verification worksheet is organized into three sections. The first contains the initial values. The second houses the model. The last area contains the assignment itself.

Most of the algebraic functions used in the assignment are well known as are inherent functions in Excel. A very useful one for business application is ROUND, which precludes highly fractionalized numbers that do not work as monetary units. In addition, the developer may need to design proprietary functions such as would control choices between discrete and continuous selection, and uniform or normal distribution shapes.

The AI constructs utilize the familiar Boolean functions in Excel (i.e., AND, OR, IF, NOT). These constructs insure the accomplishment of particular result vectors (company profitability, individual product results). Here mean values and ranges for particular spreadsheet cells are specified.

\section{Assignment Validation Worksheet}

Moving from the data to an assignment requires that a particular set of numbers that satisfy the constraints become permanently associated with a particular student. Although this can be accomplished in a variety of ways, student identification numbers that seed a random number generator appears to be an efficient strategy. Background programming also associates a student's name to be associated with that number, thus enabling the same numbers to be generated without the number. Entering any of this information calls a subroutine: Private Sub Worksheet_Change (ByVal Target As Range). This is a VBA procedure that evaluates the algebra subject to the AI constructs and produces an assignment.

The Assignment Validation worksheet also includes an assignment system control button. This activates a drop-down menu that specifies the means of distribution (email, print) and whether or not the assignment should include hints and answers. This allows the instructor to make work on the problem more convenient, control its difficulty and provide timely feedback. 


\section{Iteration and Pivot Table Worksheets}

The validation of the programming logic requires the test of repetition. Automatically entering every possible ID value between 0 and 9999, produces a worksheet that contains a column for every category and 10,001 rows, the last 10,000 of which will contain the values for each case generated. In addition, the array can be programmed to include a column for processing time, and category of results and processing method.

The large amount of data created by the iterations would be difficult to meaningfully inspect, especially if the number of columns are also large. However, such an evaluation is necessary to ensure that no student will be given an assignment that does not function as intended.

Pulling pivot tables to assess the data should start with processing time. Scenarios that cannot be generated for students in a reasonable amount of time test user patience. Descriptive statistics to evaluate production efficiency can be evaluated by requesting tables that count a progressive array of speeds. This enables the computation of cumulative frequency and can be benchmarked for acceptability.

Since the purpose of customizing assignments is to render a meaningful spread of scenarios, an assessment of their realized distribution should be made. This should be done along the lines of critical variation in the assignment. Pivot tables allow the data to be sliced in many ways for this objective. Even over 10,000 iterations, no guarantees exist that a uniform distribution will result. The instructor/developer must decide how much of a departure from that standard is acceptable.

\section{DISCUSSION}

This paper argues that the technology employed by the distance learning assignment is a reason that the method has not realized its full potential as pedagogy. Toward this objective, more advanced delivery methodologies are necessary. Most of the information systems attention in distance learning has been garnered by the pipeline that links participants and the course platform that regularizes and facilitates interaction. This paper returns attention to what most now think as mundane spreadsheet software. Although the implementation of spreadsheets in the late 1980s prevents us from asserting that no major change in the delivery of the quantitative components of business education, much more can be done with programs such as EXCEL, including work that will enhance the academic integrity of distance learning.

AI systems enable the singularity of the case solution to no longer be a constraint to education. When all students have different values, the instructor will be challenged to abstract from the numerical specifics of the case that which is most generalizable and of greatest value. The valueadded that faculty bring to distance learning will have to be much more than the mere possession of the right answers. Freed from the idea of a singular answer, students might also find creative solutions to business management dilemmas.

Despite the mere possibility of distance learning itself, the idea that the computer has revolutionized business education has not permeated the actual conduct of instruction and evaluation. Now that so much processing capabilities exist within the grasp of students, it is essential that this be included, as a major element of the pedagogy of the future. Using AI systems, the computer aids in the administration of the cases. The computer can easily be asked 
to tailor-make the numerical values of cases that will allow both the student and the instructor to be honest. In this way, cases can be substantially improved as a learning device.

The processing of the cases that we have illustrated above is practical for student use. Unlike the simulations reported in the early literature that took many hours to run on mainframe computers, these solutions usually can be processed in seconds with today's machines. These times are well within the range of acceptability for a variety of projects that might be used in distance learning. No special software is required that does not come bundled with the typical computer. Microsoft Excel is used for all work shown in this paper.

Heavily quantified assignments are quite "old bottles" in the education of managers. We believe that they still are very serviceable as pedagogical tools that bring the classroom closer to the realities of professional practice. The not-so-hidden agenda of such a level of processing sophistication is to remind students that their investment in understanding spreadsheet software to the fullest will be rewarded in most careers. However, just as modern business continues to require reinvention, the cases that model business decisions require periodic reinvigoration. Although relevant content is critical, we also must make sure that students are not denying themselves the experience of "working the numbers." Together, the AI system capabilities of current spreadsheet software can be a very powerful "new wine" for these purposes.

Artificial intelligence is the ability of computers to do things that people would say require intelligence. Let us not gainsay the intelligence required to prepare a good management case and the associated solution. Because a professor can take hours to prepare the case and the solution doing many clones manually is not such a good use of a scarce resource. Computers can do this work independently of professors. We have demonstrated that the computer can be made to show behavior indicative of intelligence, comparable or even superior to that of business professors. Using this capability, the distance learning experience can be enhanced.

\section{REFERENCES}

1. Edwards, A. and Connell, N. (1989). Expert Systems in Accounting. London: Prentice Hall.

2. Goldwater, P. and Fogarty, T. (1993). The Development of an Expert System for Accounting Education. International Journal of Applied Expert Systems, (3), 41-58.

3. Hoover, E. (2004).Students Study Less than Expected. Chronicle of Higher Education, 60(14), A1, A31.

4. Lord, A. and Melvin, K. (1997).The Attitudes of Accounting Students, Faculty and Employers Toward Cheating. Research on Accounting Ethics, (3), 1-20.

5. McCabe, D. and Trevino, L. (1996). What We Know About Cheating in College. Change, (January/February), 29-33.

6. McDermott, D. (1983).Under what conditions can a machine attribute meanings to symbols? International Joint Conferences on Artificial Intelligence, (8), 45-46.

7. West, T., Ravenscroft, S. \& Shrader, B. (2004). Cheating and Moral Judgment in the College Classroom: A Natural Experiment. Journal of Business Ethics, (54), 173-183.

8. Woods, W. (1983). Under what conditions can a machine use symbols with meanings? International Joint Conferences on Artificial Intelligence,(8), 45-46.

9. Zernike, K. (2002). With Cheating on the Rise, More Students Turn Toward Honor Codes. New York Times, (November 4), 04. 